\title{
The spread of Tembusu virus in China from 2010 to 2019
}

\author{
Yong Wang ${ }^{1}$, Yongqiu Cui ${ }^{1}$, Yeqiu $\mathrm{Li}^{1}$, and Yongdong $\mathrm{Li}^{2}$ \\ ${ }^{1}$ Anhui Agricultural University \\ ${ }^{2}$ Ningbo Municipal Center for Disease Control and Prevention
}

September 2, 2020

\begin{abstract}
Tembusu virus (TMUV) is a positive-sense RNA virus that is associated with severe reduction in egg production and even death in ducks. TMUV infection shows high incidence and is a threat to the global duck industry. However, the possible origin, genotype, and codon usage bias of TMUV are not very clear. Here, we addressed these questions by analyzing the available genomic sequences from China. The results showed that the ancestor of avian TMUV was most likely a mosquito TMUV. Moreover, three TMUV clades were identified by three different phylogenetic analysis methods. The TMUV genome exhibits a stronger mutation pressure than natural selection pressure. TMUV has the potential to cause an infection outbreak among humans, thereby posing a major threat to public health. Our findings provide important insights that reveal the ongoing TMUV spread in China and can aid in future prevention and control.
\end{abstract}

\section{Introduction}

China has a wide range of duck farming industries and has been a global leader in commercial duck production. However, with the rapid development of large-scale industries and increase in stocking density, infectious disease of ducks, especially those caused by the avian influenza virus (AIV), Newcastle disease virus (NDV), Tembusu Virus (TMUV), and duck circovirus (DuCV) have become increasingly frequent (Fan et al., 2014; L. Xie et al., 2012; Z. Xie et al., 2013; Yun et al., 2012). These infectious diseases not only cause severe losses in the duck industry, but also affect the safety of human life, such as in case of AIV and TMUV infections (Cao et al., 2011; S. Su et al., 2015).

TMUV belongs to the genus Flavivirus and family Flaviviridae. Its genome encodes three structural proteins and seven nonstructural proteins (Bai et al., 2013; Mackenzie \& Williams, 2009; Zhao et al., 2015). TMUV was first isolated in 1955 in Kuala, Malaysia, and was reported in China in 2010 (Platt et al., 1975; J. Su et al., 2011; Yun et al., 2012). The clinical symptoms caused by TMUV include depression, thin body condition, severely reduced egg production, severe neurological signs, and even death (Ti et al., 2015; Zhu et al., 2012). Since 2010, TMUV has become one of the most economically important infectious diseases of ducks in China (Wang et al., 2011; Yu et al., 2013).

As a member of the genus Flavivirus, the relevance of TMUV to human and animals is unclear (Zhang, Chen, Mahalingam, Wang, \& Cheng, 2017). However, other members of Flavivirus such as West Nile virus (WNV), dengue virus (DENV), yellow fever virus (YFN), Japanese encephalitis virus (JEV), and Zika virus cause serious diseases in humans, including mild febrile symptoms and fatal hemorrhagic or neurologic disease every year worldwide (Q. Chen et al., 2020; Gubler, 2002; Hayes et al., 2005; Lanciotti et al., 1999). In nature, birds usually serve as amplifying hosts, including for WNV, Sitiawan virus, Usutu virus, and Bagaza virus. Notably, there has been a survey reporting evidence that TMUV has the potential to be transmitted to humans by infected birds, emphasizing the need for further study of the virus (Aguero et al., 2011; Barnard, Buys, Du Preez, Greyling, \& Venter, 1980; Becker et al., 2012; Gould \& Solomon, 2008; Kono et al., 2000; Niu et al., 2018; Weissenbock et al., 2002; Zhang et al., 2017). 
In this study, we obtained the TMUV gene sequences reported across China from NCBI. Then, the epidemiologic, genetic diversity, and evolutionary dynamics of TMUV were analyzed through bioinformatics research methods. The findings of this study are expected to aid in the prevention and control of TMUV in the future.

\section{Materials and methods}

\section{Sequence datasets}

All sequences used in this study were obtained from the GenBank database of NCBI (https://www.ncbi.nlm.nih.gov/). A total of 40 strains of avian TMUV, including duck TMUV, goose TMUV, and chicken TMUV were obtained. Furthermore, a total of 4 related viral strains were also obtained from GenBank, including 4 strains of mosquito TMUV, 2 strains of Zika virus, 1 strain of WNV, and 1 strain of DENV. The details of these sequences are presented in Table 1.

\section{Tracking the origin of TMUV}

Since the first report of TMUV in China, it has spread quickly. However, until now, the exact origin has not been elucidated. To investigate the origin of TMUV, a maximum likelihood (ML) tree was constructed using open reading frame $(\mathrm{ORF})$ gene sequences. Furthermore, to verify the result of the ML tree, a maximum clade credibility (MCC) tree was constructed with the complete ORFs.

\section{Epidemiology of TMUV}

To investigate the phylogeny trend of TMUV, 40 strains were obtained from GenBank, and were used as representative strains of the complete genome of TMUV obtained from mainland China from 2010 to 2019. All 40 sequences were aligned using ClustalW, which was applied in MEGA 6. The best substitution model was selected by jModelTest according to the Bayesian information criterion (BIC) score. Furthermore, to study the epidemiological trends of TMUV, a Bayesian skyline plot (BSP) was established. Additionally, to ensure the feasibility of the results, two independent runs of the chain were performed and compared to ensure adequate sampling; a chain length comprised 10,000,000 generations with sampling every 1,000 generations with $10 \%$ burn-in removed.

\section{Selection pressure and codon usage bias analysis}

To analyze the selection pressure on the ORF of TMUV, 4 different methods were used: Single Likelihood Ancestor Counting (SLAC), Fixed Effects Likelihood (FEL), Fast Unconstrained Bayesian AppRoximation for inferring selection (FUBAR), and mixed effects model of evolution (MEME) on DATAMONKEY (http://www.datamonkey.org/) (Katoh \& Standley, 2013; Murrell et al., 2013; Murrell et al., 2012; Smith et al., 2015). The basic nucleotide composition (A\%, T\%, C\%, and G\%), nucleotide at the third position of synonymous codons (A3s\%, T3s\%, C3s\%, and G3s\%), GC content on the third synonymous codon position (GC3s), and the effective number of codons (ENC) were calculated for the VP2 gene sequence using CodonW (1.4.2).

The ENC value ranged from 20 to 61 . The higher the ENC value, the smaller is the codon usage bias. Therefore, when the evaluation value is 20, the deviation is stronger. When the ENC value is less than 35, codon usage bias is considered very strong (Comeron \& Aguade, 1998; He et al., 2019). Subsequently, an ENC-plot (ENC plotted against GC3s) was drawn to reflect the factors influencing codon usage bias (Fuglsang, 2004; Wright, 1990). The expected ENC was calculated as follows ('s' is the frequency of G+ $\mathrm{C}$ at the third position of synonymous codons):

$$
\text { ENC expect }=2+s+\frac{29}{s^{2}+(1-s)^{2}}
$$

At the same time, the neutrality plot analysis was also performed, which can reflect the degree of the influence of mutation pressure and natural selection on codon use patterns. We can judge whether natural selection 
or mutation is dominant based on the slope of the curve. All data were plotted using Origin 2018 software.

\section{Results}

\section{Origin of TMUV in China}

To trace the origin of TMUV, we constructed an ML tree based on the ORF gene sequence (Fig. 1a). We found that all the avian TMUV strains were closely related to the mosquito TMUV with high confidence. The West Nile virus lineage 2 (NC001563) was selected as outgroup of the mosquito TMUV and avian TMUV strains, which might indicate a potential mosquito TMUV origin. To further verify this result, an MCC tree was constructed (Fig. 1b). The MCC tree showed a topology similar to that of the ML tree. Although this provides evidence that a close relationship exists between avian TMUV and mosquito TMUV, it is worth noting that only 3 strains of mosquito TMUV could be obtained from GenBank; we will update the analysis once more mosquito TMUV is available.

\section{TMUV genetic diversity and epidemiological data in China}

To investigate the genetic diversity of TMUV, we used three different methods (NJ, ML, and MCC) to construct the phylogenies using the ORF sequence of the TMUVs. All of the strains represent four different avian species that were isolated in China from 2010 to 2019, and the strain XZW10694 (Accession number: KY937189) as outgroup. Three different clades were observed using the three different methods (Fig.2a, $2 \mathrm{~b}$, and 2c). Compared with a previous study (Dai, Li, \& Tao, 2015), a new clade was observed in the three different phylogenetic trees. This new clade (clade 3) was isolated in Shandong in 2014 and the host was Anas platyrhynchos (Accession number: MH748542). Clade 3 showed a longer evolutionary distance compared to clades 1 and 2, which might suggest that clade 3 is a potential new genotype. Based on the BSP, the TMUV might have diversified from 2010 to 2012, after which it entered the platform period (Fig. $3)$.

\section{Selection pressure and codon usage bias analysis}

An analysis of selection pressure can be used to explore whether the existence of variability is related to selection pressure. The results showed three positive selection sites of TMUV detected by three methods, and six positive selection sites detected by two methods; details are shown in Table 2.

To investigate the evolutionary characteristics of TMUV, the ORF gene sequence was analyzed for codon usage bias. The mean value of $\mathrm{G} \%$ was the highest, followed by $\mathrm{A} \%, \mathrm{C} \%$, and $\mathrm{T} \%$, which indicates a strong compositional bias in favor of $\mathrm{G}$ or A. Furthermore, in terms of synonymous codons, we found that at the third position (A3\%, T3\%, C3\%, G3\%), the highest value was observed for nucleotide $\mathrm{G}$ for all genotypes.

At the same time, to investigate the effects of selective pressure and mutation pressure on the evolution of TMUV, an ENC-plot and neutrality plot analyses were conducted. The mean ENC value was $53.2575 \pm$ 0.458, which was higher than 35, indicating a low codon bias in TMUV. The ENC-plot and neutrality plot analyses (Fig. 4a and $4 \mathrm{~b}$ ) indicated mutation pressure rather than natural selection pressure.

\section{Discussion}

Since TMUV was first reported in 2010, it had widely spread in China (Liu et al., 2013; Z. Xie et al., 2014; Yan et al., 2017; Yun et al., 2012; X. Zhou et al., 2016; Zhu et al., 2012). Many studies have focused on the mechanism of virus action and the establishment of detection methods (Bai et al., 2015; L. Chen et al., 2019; S. Chen et al., 2018; Cui et al., 2018; Fu, Ji, Liu, Dafallah, \& Zhu, 2015; Hu et al., 2020; Jiang et al., 2012; Wu et al., 2016). In this study, we provide new insights into the origin, genetic diversity, and codon usage of TMUV. Compared with the previous studies, we provide better evidence for the origin, genotype diversity, and codon usage bias of TMUV (Dai et al., 2015; H. Zhou et al., 2015). We found that mosquito TMUV, which was isolated from 2015 to 2019, shared the most recent common ancestor with avian TMUV. Furthermore, we found a new potential clade that was isolated in the Shandong province; this new clade also showed high homology with the mosquito TMUV. Overall, the mosquito TMUV is the most likely origin of avian TMUV based on the currently available sequences. Mosquitoes are considered the main 
transmission hosts ofFlaviviridae. TMUV host jumps pose a major threat to the duck industry and public safety. According to previous research, TMUV virus has also been detected in humans (Cao et al., 2011).

Previously, Tao et al. used ML trees to type TMUV (Dai et al., 2015). In this study, we explored the phylogenetic history of TMUV isolated in China from 2010 to 2019. To identify the genotype of TMUV, three different methods (NJ, ML, and MCC) were used. All three methods showed that Chinese TMUV can be divided into three different clades (TMUV 1, TMUV 2, and TMUV 3). Additionally, within the TMUV 1 clade, 3 stable subclades were identified (TMUV 1a, TMUV 1b, and TMUV 1c), and in the TMUV 2 clade, 2 stable subclades were identified (TMUV 2a and TMUV 2b). Compared with the previous study (Dai et al., 2015; Ninvilai, Tunterak, Oraveerakul, Amonsin, \& Thontiravong, 2019), the TMUV in the current study showed more genetic diversity. Nonetheless, in recent years, the rate of emergence of new genotypes has slowed, which is also supported by the Bayesian skyline plot analysis.

The codon usage bias can be used to understand the changes in codon usage and its influencing factors during the evolution of TMUV. Gene mutation pressure, secondary protein structure, natural selection pressure, and external environment were found to be the main factors causing codon bias (Bera et al., 2017; Hershberg \& Petrov, 2008). In this study, ENC-plot analysis and neutrality plot analysis showed that mutation pressure played a decisive role in codon usage bias, which is similar to the results of previous research (H. Zhou et al., 2015). Notably, the preferred codons consisted of the optimal codons for human cells, which indicates that TMUV has the potential to infect humans after cross-species transmission; this is also supported by previous studies that have detected TMUV in humans (Tang et al., 2013; H. Zhou et al., 2015).

Cross-species transmission of viruses is a serious threat to public safety. There are already many such cases that have adversely affected public health, including SARS, MERS, SARS-CoV-2, and Zika virus infections (Dye \& Gay, 2003; Lazear, Stringer, \& de Silva, 2016; Verdoni et al., 2020; Zumla, Alagaili, Cotten, \& Azhar, 2016). TMUV has the potential to spread across species and cause great damage. With the rapidly increasing population and increasing constant contact of humans with nature, there is an increasing possibility of an interaction between different hosts (avian to mosquito to human). Therefore, it is likely not a matter of if but when TMUV will cause a major infection outbreak in the human population.

In summary, we collected the sequences of TMUV that were reported in China over the past 10 years. We assigned the possible mosquito origin of TMUV and divided the TMUV into three clades. Furthermore, we raised the possibility that in future TMUV may infect people and cause serious consequences. Future research should focus on the following aspects: recombinant TMUV in animals, cross-species transmission of TMUV within the animal, and finally, establishing a detection network to prevent the sudden emergence of TMUV with high virulence that can infect humans. Overall, our study characterized TMUV spread in China and provided insights that may aid in future prevention and control of the virus spread.

\section{Data Availability Statement}

The data used to support the findings of this study are included within the article.

\section{Declaration of conflicting interests}

The authors declare that they have no conflict of interest.

\section{Acknowledgments}

This study was supported by the Ningbo Health Branding Subject Fund (No. ppxk2018-10), key research project of national science and technology (No. 2016YFD0501003).

\section{Compliance with Ethical Standards}

All experiments were compliant with the ethical standards of Anhui Agricultural University.

\section{References}


Aguero, M., Fernandez-Pinero, J., Buitrago, D., Sanchez, A., Elizalde, M., San Miguel, E., ... JimenezClavero, M. A. (2011). Bagaza virus in partridges and pheasants, Spain, 2010. Emerg Infect Dis, 17 (8), 1498-1501. doi:10.3201/eid1708.110077

Bai, X., Lv, R., Liu, C., Qiu, N., He, Y., Yin, X., . . Zhang, Y. (2013). Molecular characterization of a duck Tembusu virus from China. Virus Genes, 47 (3), 478-482. doi:10.1007/s11262-013-0966-3

Bai, X., Shaozhou, W., Zhang, Q., Li, C., Qiu, N., Meng, R., . . Zhang, Y. (2015). Characterization of monoclonal antibodies against duck Tembusu virus E protein: an antigen-capture ELISA for the detection of Tembusu virus infection. Arch Virol, 160 (3), 757-764. doi:10.1007/s00705-014-2312-z

Barnard, B. J., Buys, S. B., Du Preez, J. H., Greyling, S. P., \& Venter, H. J. (1980). Turkey meningoencephalitis in South Africa. Onderstepoort J Vet Res, 47 (2), 89-94.

Becker, N., Jost, H., Ziegler, U., Eiden, M., Hoper, D., Emmerich, P., . . Schmidt-Chanasit, J. (2012). Epizootic emergence of Usutu virus in wild and captive birds in Germany. PLoS One, 7 (2), e32604. doi:10.1371/journal.pone.0032604

Bera, B. C., Virmani, N., Kumar, N., Anand, T., Pavulraj, S., Rash, A., . . . Tripathi, B. N. (2017). Genetic and codon usage bias analyses of polymerase genes of equine influenza virus and its relation to evolution. BMC Genomics, 18 (1), 652. doi:10.1186/s12864-017-4063-1

Cao, Z., Zhang, C., Liu, Y., Liu, Y., Ye, W., Han, J., . . Zhang, D. (2011). Tembusu virus in ducks, china. Emerg Infect Dis, 17 (10), 1873-1875. doi:10.3201/eid1710.101890

Chen, L., Yu, B., Hua, J., Ni, Z., Ye, W., Yun, T., \& Zhang, C. (2019). Optimized Expression of Duck Tembusu Virus E Gene Delivered by a Vectored Duck Enteritis Virus In Vitro. Mol Biotechnol, 61 (10), 783-790. doi:10.1007/s12033-019-00206-1

Chen, Q., Gouilly, J., Ferrat, Y. J., Espino, A., Glaziou, Q., Cartron, G., . . . Jabrane-Ferrat, N. (2020). Metabolic reprogramming by Zika virus provokes inflammation in human placenta. Nat Commun, 11 (1), 2967. doi:10.1038/s41467-020-16754-Z

Chen, S., He, Y., Zhang, R., Liu, P., Yang, C., Wu, Z., . . Cheng, A. (2018). Establishment of a reverse genetics system for duck Tembusu virus to study virulence and screen antiviral genes. Antiviral Res, 157, 120-127. doi:10.1016/j.antiviral.2018.06.016

Comeron, J. M., \& Aguade, M. (1998). An evaluation of measures of synonymous codon usage bias. J Mol Evol, 47 (3), 268-274. doi:10.1007/pl00006384

Cui, M., Jia, R., Huang, J., Wu, X., Hu, Z., Zhang, X., . . . Cheng, A. (2018). Analysis of the microRNA expression profiles in DEF cells infected with duck Tembusu virus. Infect Genet Evol, 63 , 126-134. doi:10.1016/j.meegid.2018.05.020

Dai, L., Li, Z., \& Tao, P. (2015). Evolutionary analysis of Tembusu virus: evidence for the emergence of a dominant genotype. Infect Genet Evol, 32 , 124-129. doi:10.1016/j.meegid.2015.03.004

Dye, C., \& Gay, N. (2003). Epidemiology. Modeling the SARS epidemic.Science, 300 (5627), 1884-1885. doi:10.1126/science.1086925

Fan, S., Zhou, L., Wu, D., Gao, X., Pei, E., Wang, T., . . Xia, X. (2014). A novel highly pathogenic H5N8 avian influenza virus isolated from a wild duck in China. Influenza Other Respir Viruses, 8 (6), 646-653. doi:10.1111/irv.12289

Fu, Y., Ji, Y., Liu, B., Dafallah, R. M., \& Zhu, Q. (2015). Development of a solid-phase competition ELISA to detect antibodies against newly emerged Duck Tembusu virus. J Virol Methods, 224 , 73-76. doi:10.1016/j.jviromet.2015.08.017 
Fuglsang, A. (2004). The 'effective number of codons' revisited.Biochem Biophys Res Commun, 317 (3), 957-964. doi:10.1016/j.bbrc.2004.03.138

Gould, E. A., \& Solomon, T. (2008). Pathogenic flaviviruses.Lancet, 371 (9611), 500-509. doi:10.1016/S01406736(08)60238-X

Gubler, D. J. (2002). The global emergence/resurgence of arboviral diseases as public health problems. Arch Med Res, 33 (4), 330-342. doi:10.1016/s0188-4409(02)00378-8

Hayes, E. B., Sejvar, J. J., Zaki, S. R., Lanciotti, R. S., Bode, A. V., \& Campbell, G. L. (2005). Virology, pathology, and clinical manifestations of West Nile virus disease. Emerg Infect Dis, 11 (8), 1174-1179. doi:10.3201/eid1108.050289b

He, W., Zhao, J., Xing, G., Li, G., Wang, R., Wang, Z., . . Zhou, J. (2019). Genetic analysis and evolutionary changes of Porcine circovirus 2. Mol Phylogenet Evol, 139 , 106520. doi:10.1016/j.ympev.2019.106520

Hershberg, R., \& Petrov, D. A. (2008). Selection on codon bias.Annu Rev Genet, 42 , 287-299. doi:10.1146/annurev.genet.42.110807.091442

Hu, Z., Pan, Y., Cheng, A., Zhang, X., Wang, M., Chen, S., . . Jia, R. (2020). Autophagy Promotes Duck Tembusu Virus Replication by Suppressing p62/SQSTM1-Mediated Innate Immune Responses In Vitro. Vaccines (Basel), 8 (1). doi:10.3390/vaccines8010022

Jiang, T., Liu, J., Deng, Y. Q., Su, J. L., Xu, L. J., Liu, Z. H., . . Qin, C. F. (2012). Development of RTLAMP and real-time RT-PCR assays for the rapid detection of the new duck Tembusu-like BYD virus.Arch Virol, 157 (12), 2273-2280. doi:10.1007/s00705-012-1431-7

Katoh, K., \& Standley, D. M. (2013). MAFFT multiple sequence alignment software version 7: improvements in performance and usability. Mol Biol Evol, 30 (4), 772-780. doi:10.1093/molbev/mst010

Kono, Y., Tsukamoto, K., Abd Hamid, M., Darus, A., Lian, T. C., Sam, L. S., . . Narita, M. (2000). Encephalitis and retarded growth of chicks caused by Sitiawan virus, a new isolate belonging to the genus Flavivirus. Am J Trop Med Hyg, 63 (1-2), 94-101. doi:10.4269/ajtmh.2000.63.94

Lanciotti, R. S., Roehrig, J. T., Deubel, V., Smith, J., Parker, M., Steele, K., . . Gubler, D. J. (1999). Origin of the West Nile virus responsible for an outbreak of encephalitis in the northeastern United States. Science, 286 (5448), 2333-2337. doi:10.1126/science.286.5448.2333

Lazear, H. M., Stringer, E. M., \& de Silva, A. M. (2016). The Emerging Zika Virus Epidemic in the Americas: Research Priorities. JAMA, 315 (18), 1945-1946. doi:10.1001/jama.2016.2899

Liu, P., Lu, H., Li, S., Wu, Y., Gao, G. F., \& Su, J. (2013). Duck egg drop syndrome virus: an emerging Tembusu-related flavivirus in China.Sci China Life Sci, 56 (8), 701-710. doi:10.1007/s11427-013-4515-z

Mackenzie, J. S., \& Williams, D. T. (2009). The zoonotic flaviviruses of southern, south-eastern and eastern Asia, and Australasia: the potential for emergent viruses. Zoonoses Public Health, 56 (6-7), 338-356. doi:10.1111/j.1863-2378.2008.01208.x

Murrell, B., Moola, S., Mabona, A., Weighill, T., Sheward, D., Kosakovsky Pond, S. L., \& Scheffler, K. (2013). FUBAR: a fast, unconstrained bayesian approximation for inferring selection. Mol Biol Evol, 30 (5), 1196-1205. doi:10.1093/molbev/mst030

Murrell, B., Wertheim, J. O., Moola, S., Weighill, T., Scheffler, K., \& Kosakovsky Pond, S. L. (2012). Detecting individual sites subject to episodic diversifying selection. PLoS Genet, 8 (7), e1002764. doi:10.1371/journal.pgen.1002764

Ninvilai, P., Tunterak, W., Oraveerakul, K., Amonsin, A., \& Thontiravong, A. (2019). Genetic characterization of duck Tembusu virus in Thailand, 2015-2017: Identification of a novel cluster. Transbound Emerg Dis, 66 (5), 1982-1992. doi:10.1111/tbed.13230 
Niu, X., Wang, H., Wei, L., Zhang, M., Yang, J., Chen, H., . . Diao, Y. (2018). Epidemiological investigation of H9 avian influenza virus, Newcastle disease virus, Tembusu virus, goose parvovirus and goose circovirus infection of geese in China. Transbound Emerg Dis, 65 (2), e304-e316. doi:10.1111/tbed.12755

Platt, G. S., Way, H. J., Bowen, E. T., Simpson, D. I., Hill, M. N., Kamath, S., . . Heathcote, O. H. (1975). Arbovirus infections in Sarawak, October 1968-February 1970 Tembusu and Sindbis virus isolations from mosquitoes. Ann Trop Med Parasitol, 69 (1), 65-71. doi:10.1080/00034983.1975.11686984

Smith, M. D., Wertheim, J. O., Weaver, S., Murrell, B., Scheffler, K., \& Kosakovsky Pond, S. L. (2015). Less is more: an adaptive branch-site random effects model for efficient detection of episodic diversifying selection. Mol Biol Evol, 32 (5), 1342-1353. doi:10.1093/molbev/msv022

Su, J., Li, S., Hu, X., Yu, X., Wang, Y., Liu, P., . . Gao, G. F. (2011). Duck egg-drop syndrome caused by BYD virus, a new Tembusu-related flavivirus. PLoS One, 6 (3), e18106. doi:10.1371/journal.pone.0018106

Su, S., Bi, Y., Wong, G., Gray, G. C., Gao, G. F., \& Li, S. (2015). Epidemiology, Evolution, and Recent Outbreaks of Avian Influenza Virus in China. J Virol, 89 (17), 8671-8676. doi:10.1128/JVI.01034-15

Tang, Y., Gao, X., Diao, Y., Feng, Q., Chen, H., Liu, X., . . Yu, C. (2013). Tembusu virus in human, China. Transbound Emerg Dis, 60 (3), 193-196. doi:10.1111/tbed.12085

Ti, J., Zhang, L., Li, Z., Zhao, D., Zhang, Y., Li, F., \& Diao, Y. (2015). Effect of age and inoculation route on the infection of duck Tembusu virus in Goslings. Vet Microbiol, 181 (3-4), 190-197. doi:10.1016/j.vetmic.2015.10.001

Verdoni, L., Mazza, A., Gervasoni, A., Martelli, L., Ruggeri, M., Ciuffreda, M., . . . D’Antiga, L. (2020). An outbreak of severe Kawasaki-like disease at the Italian epicentre of the SARS-CoV-2 epidemic: an observational cohort study. Lancet, 395 (10239), 1771-1778. doi:10.1016/S0140-6736(20)31103-X

Wang, Y., Yuan, X., Li, Y., Yu, K., Yang, J., Xu, H., . . Qin, Z. (2011). Rapid detection of newly isolated Tembusu-related Flavivirus by reverse-transcription loop-mediated isothermal amplification assay. Virol J, 8 , 553. doi:10.1186/1743-422X-8-553

Weissenbock, H., Kolodziejek, J., Url, A., Lussy, H., Rebel-Bauder, B., \& Nowotny, N. (2002). Emergence of Usutu virus, an African mosquito-borne flavivirus of the Japanese encephalitis virus group, central Europe. Emerg Infect Dis, 8 (7), 652-656. doi:10.3201/eid0807.020094

Wright, F. (1990). The 'effective number of codons' used in a gene.Gene, 87 (1), 23-29. doi:10.1016/03781119(90)90491-9

Wu, X., Shi, Y., Yan, D., Li, X., Yan, P., Gao, X., . . . Li, Z. (2016). Development of a PCR-Based Reverse Genetics System for an Attenuated Duck Tembusu Virus Strain. PLoS One, 11 (6), e0156579. doi:10.1371/journal.pone.0156579

Xie, L., Xie, Z., Zhao, G., Liu, J., Pang, Y., Deng, X., . . Fan, Q. (2012). Complete genome sequence analysis of a duck circovirus from Guangxi pockmark ducks. J Virol, 86 (23), 13136. doi:10.1128/JVI.02494-12

Xie, Z., Xie, L., Xu, Z., Liu, J., Pang, Y., Deng, X., . . . Luo, S. (2013). Identification of a Genotype IX Newcastle Disease Virus in a Guangxi White Duck. Genome Announc, 1 (5). doi:10.1128/genomeA.00836-13

Xie, Z., Zeng, T., Xie, L., Deng, X., Xie, Z., Liu, J., . . . Luo, S. (2014). Genome Analysis of a Tembusu Virus, GX2013H, Isolated from a Cheery Valley Duck in Guangxi, China. Genome Announc, 2 (4). doi:10.1128/genomeA.00466-14

Yan, Z., Shen, H., Wang, Z., Lin, W., Xie, Q., Bi, Y., \& Chen, F. (2017). Isolation and Characterization of a Novel Tembusu Virus Circulating in Muscovy Ducks in South China. Transbound Emerg Dis, 64 (5), e15-e17. doi:10.1111/tbed.12525 
Yu, K., Sheng, Z. Z., Huang, B., Ma, X., Li, Y., Yuan, X., . . Sun, H. (2013). Structural, antigenic, and evolutionary characterizations of the envelope protein of newly emerging Duck Tembusu Virus. PLoS One, 8 (8), e71319. doi:10.1371/journal.pone.0071319

Yun, T., Zhang, D., Ma, X., Cao, Z., Chen, L., Ni, Z., . . Zhang, C. (2012). Complete genome sequence of a novel flavivirus, duck tembusu virus, isolated from ducks and geese in china. J Virol, 86 (6), 3406-3407. doi:10.1128/JVI.07132-11

Zhang, W., Chen, S., Mahalingam, S., Wang, M., \& Cheng, A. (2017). An updated review of avian-origin Tembusu virus: a newly emerging avian Flavivirus. J Gen Virol, 98 (10), 2413-2420. doi:10.1099/jgv.0.000908

Zhao, D., Huang, X., Han, K., Liu, Y., Yang, J., Liu, Q., . . Li, Y. (2015). Protective immune response against newly emerging goose tembusu virus infection induced by immunization with a recombinant envelope protein. Lett Appl Microbiol, 61 (4), 318-324. doi:10.1111/lam.12459

Zhou, H., Yan, B., Chen, S., Wang, M., Jia, R., \& Cheng, A. (2015). Evolutionary characterization of Tembusu virus infection through identification of codon usage patterns. Infect Genet Evol, 35 , 27-33. doi:10.1016/j.meegid.2015.07.024

Zhou, X., Zhang, T., Song, D., Huang, T., Peng, Q., Chen, Y., . . . Tang, Y. (2016). Whole-Genome Sequence of Duck Tembusu Virus Strain DTMUV/CH/2014, Isolated in China. Genome Announc, 4 (1). doi:10.1128/genomeA.01657-15

Zhu, W., Chen, J., Wei, C., Wang, H., Huang, Z., Zhang, M., . . . Su, S. (2012). Complete genome sequence of duck Tembusu virus, isolated from Muscovy ducks in southern China. J Virol, 86 (23), 13119. doi:10.1128/JVI.02361-12

Zumla, A., Alagaili, A. N., Cotten, M., \& Azhar, E. I. (2016). Infectious diseases epidemic threats and mass gatherings: refocusing global attention on the continuing spread of the Middle East Respiratory syndrome coronavirus (MERS-CoV). BMC Med, 14 (1), 132. doi:10.1186/s12916-016-0686-3

\section{Figure captions}

Fig. 1 The origin of Tembusu virus (TMUV) was deduced using the complete ORF gene. a) Maximum likelihood tree reconstructed with MEGA 6.0 using different species of TMUV. b) Maximum clade credibility tree reconstructed using BEAST 2.0.

Fig. 2 Phylogenetic analysis of Tembusu virus (TMUV). a) Maximum likelihood tree reconstructed using MEGA 6.0. b) Neighbor-Joining phylogenetic tree reconstructed using MEGA 6.0. c) Maximum clade credibility tree reconstructed using GTR $+\mathrm{I}+\mathrm{G}$ substation model and with an uncorrected relaxed clock (lognormal) of TMUV complete ORF gene sequence. The different genotypes are shown using different colors.

Fig. 3 Bayesian skyline plot of Tembusu virus (TMUV). The genetic diversity of TMUV is shown on the y-axis with $95 \%$ highest posterior density (HPD) shown in blue.

Fig. 4 Effective number of codons (ENC)-plot analysis and neutrality plot of TMUV complete genome. a) ENC-plot analysis show that the observed ENC values were below the expected curve regardless of genotype. b) Neutrality plot analysis indicated that the correlations between GC12 and GC3 were not significant in the Tembusu virus (TMUV) genotypes. 

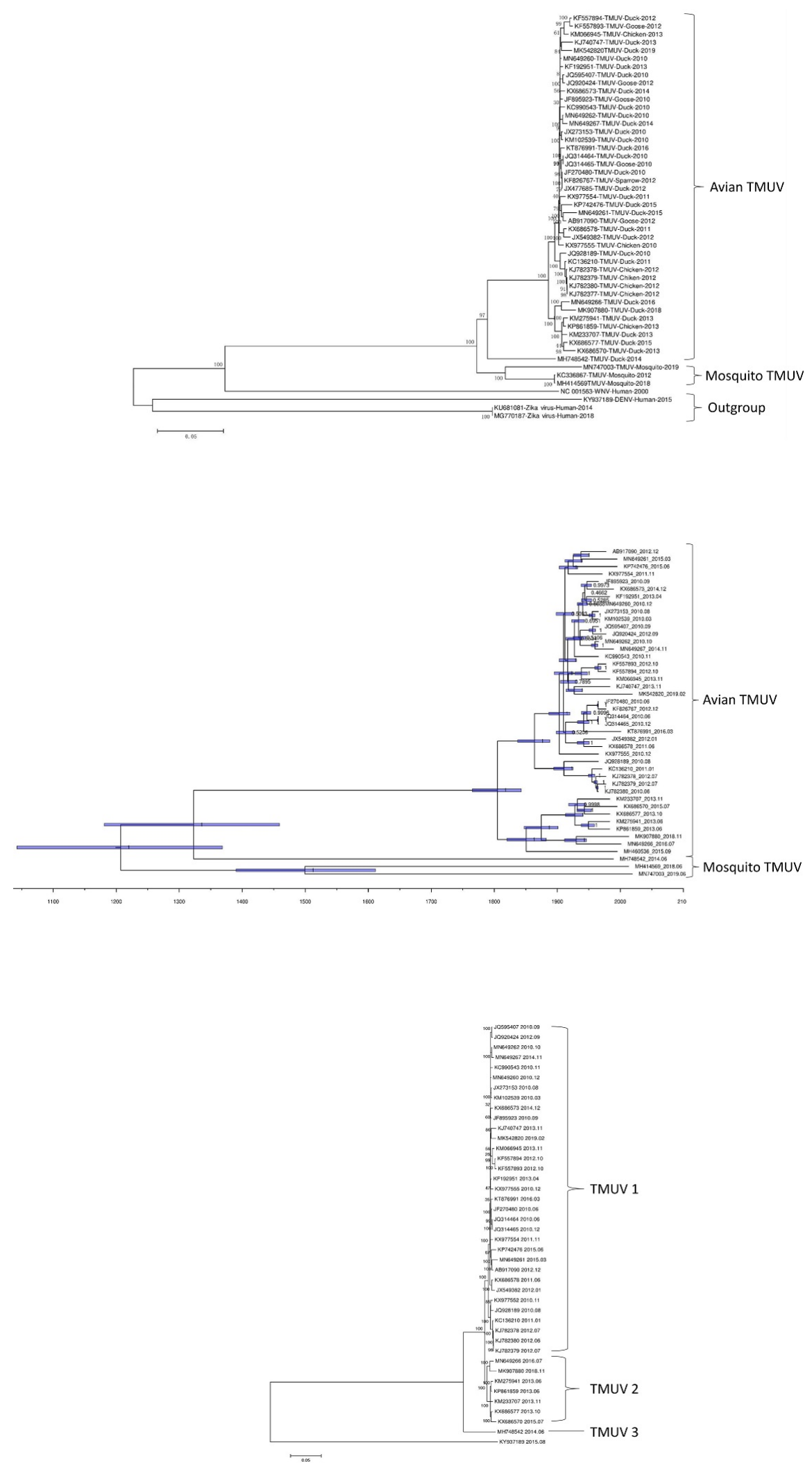


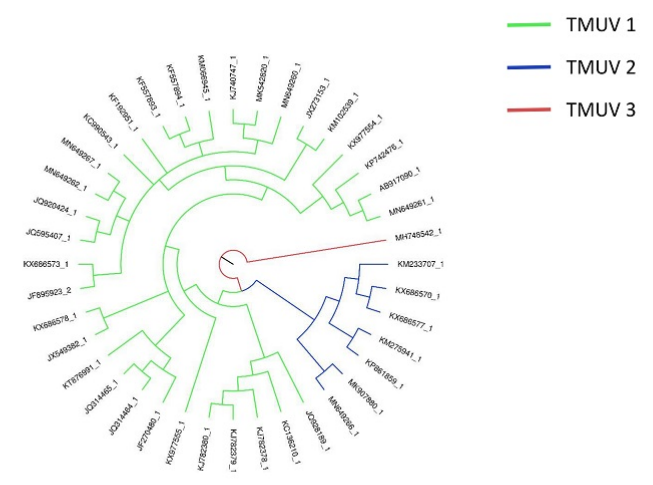

$\overline{1.1}$

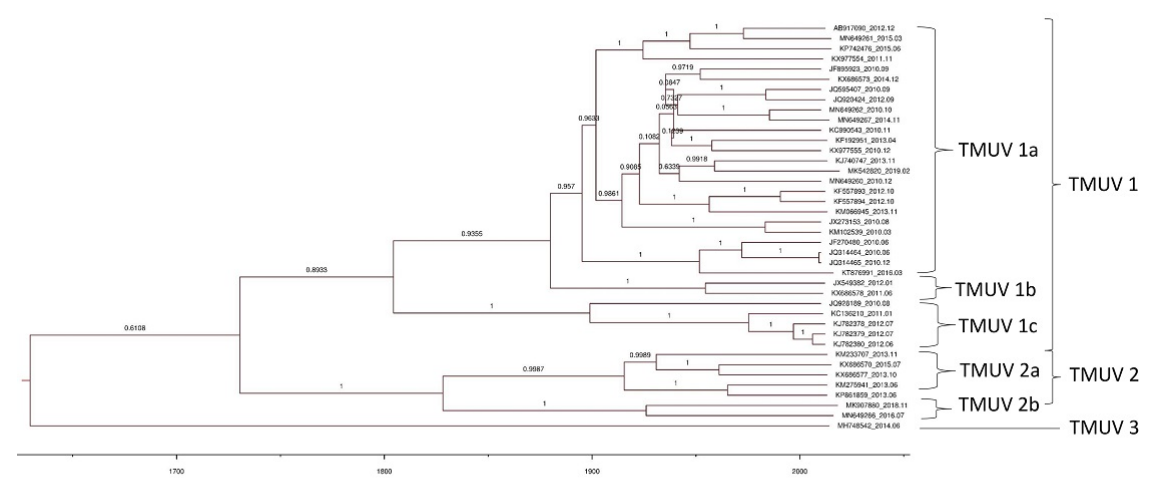



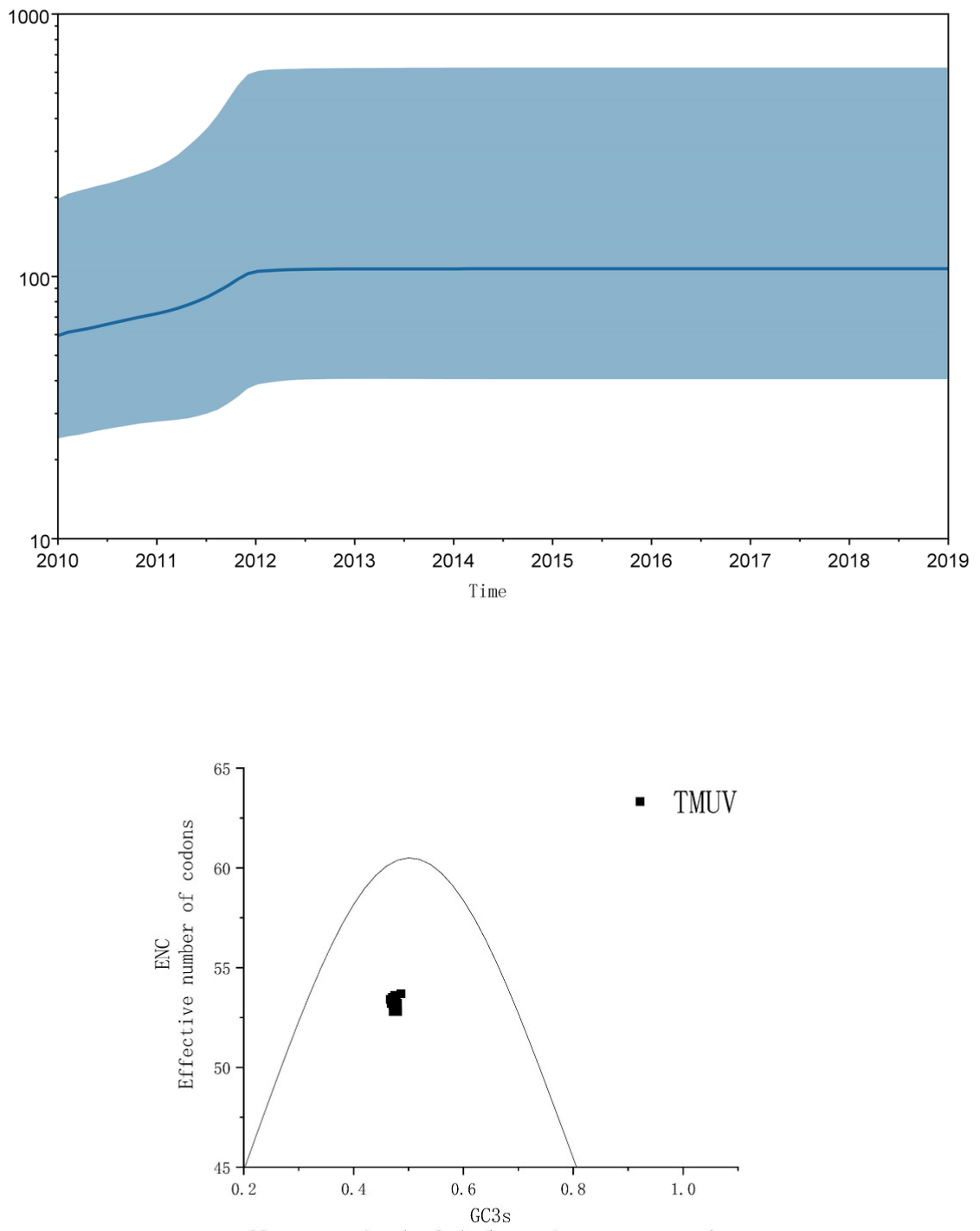

GC content in the $3 \mathrm{rd}$ place of synonymous codon 


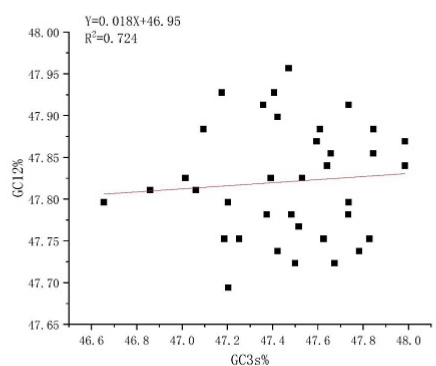

